\title{
YAKKA: UN CASTILLO RURAL DE LA CORA DE MURCIA. SIGLOS XI AL XIII ESTRUCTURA ADMINISTRATIVA Y POBLAMIENTO
}

Por

LIBORIO RUIZ MOLINA 
El presente estudio pretende exponer una serie de sugerencias a modo de hipótesis inicial en los trabajos de investigación sobre Yecla en época musulmana.

El campo de la arqueología, de reciente introducción en el terreno de la Edad Media, abre unas enormes posibilidades en las nuevas tendencias historiográficas. Esta vía abierta hace unos años por Andrée Bazzana ha hecho cambiar formas y hábitos en el proceder, al acometer estudios históricos sobre un período hasta hace dos décadas prácticamente desconocido.

La ruptura de esa barrera en el tiempo fijada en la "Reconquista* como punto de partida en la historia medieval regional, ha venido propiciada por la técnica arqueológica, que nos ha servido y sirve para conocer con mayor precisión la cultura material en el marco cronológico de los siglos VIII al XV, y complementarse así con los estudios históricos basados en los análisis de las fuentes documentales escritas, tanto árabes como cristianas. De ello se desprende que en lo sucesivo no podremos concebir los trabajos de investigación sin conseguir una simbiosis entre ambos componentes.

Así pues, procuraremos dar una aproximación, en base a los datos que poseemos hasta la fecha, a la estructura administrativa dentro del marco 
regional, al marco físico propiamente dicho y sus límites territoriales, y las relaciones con las comarcas circundantes a través de las vías de comunicación, trabajando pues en el ámbito de la geografía histórica, donde no olvidaremos algunos apuntes sobre el poblamiento.

Los datos que las fuentes árabes escritas de los siglos XI al XIII se refieren a Yecla son muy escuetos, aunque no obstante nos van a permitir trabajar y cimentar las tesis iniciales para futuros trabajos.

Ibn Sa'ĩd indicaba que Yakka era un hișn de Murcia de donde era originario el poeta $\mathrm{Abu}$ Bakr Yahya Shal al-Yakkĩ. Estaríamos por tanto ante el primer concepto de estructura poblacional, es decir, el hișn o castillo rural, así definido por Bazzana, Cressier y Guichard (1), al objeto de poder diferenciarlo de otras formas de habitat: madina (ciudad), qal'a (alcazaba), qarya (alquería), balda (poblado), etc... Para ellos, los hūsūn, se constituyen en centros de organización socio-política con un cierto grado autónomo, presentes desde los primeros tiempos de la conquista. (s. VIII) Cabría pues plantearnos si este sistema de habitat o base organizativa de población es una aportación musulmana o si por el contrario se trata de un modo o forma de asentamiento auctóctono. Todo parece indicar esta segunda consideración. En el caso de Yecla y las comarcas circundantes a ella se muestra como un hecho tangible. Las poblaciones indígenas preromanas se organizan en torno a una fortaleza o poblado fortificado en altura, situado en lugares geográficamente estratégicos de control de las llanuras próximas. La conquista romana, y la posterior organización admi-

(1) BAZZANA, Andrée; CRESSIER, Patrice; GUICHARD, Pierre. Les Chateaux Ruraux d'AlAndalus. Histoire et Archeologie des busun du sud-est de l'Espagne. Publications de la Casa Velazquez. Serie Archeologie, XI. Madrid, 1988. 
nistrativa de las áreas rurales con establecimientos agrícolas en llano (villas rústicas), rompió con la forma tradicional de poblamiento. A partir del siglo $\mathrm{V} \mathrm{d}$. C. puede observarse una reocupación de los habitats en altura, con un claro carácter de autodefensa. Ejemplos los tenemos en el Castillo de Santa Bárbara (Alicante), La Moleta (Elche), el Castillo del Río (Aspe), Monastil (Elda), El Castellar (Alcoy), el Castell de Castalla, Begastri (Cehegín) y muy probablemente el propio Cerro del Castillo en Yecla (2).

Este será el paisaje poblacional que encontrarán los musulmanes en el momento de la conquista, en algunos casos como Begastri, Orihuela o Elche con un cierto desarrollo urbano y una óptima estructura socio-política bajo la fórmula del obispado. (Begastri, Elche) Así pues, aquí es donde debemos buscar el origen de los hūsūn, por lo que deberemos abandonar esa idea que hasta hace poco tiempo compartíamos, por lo menos para el área del Vinalopó, de que la instalación de estos castillos era un fenómeno propio de los siglos XI y XII con el carácter de reforzamiento de fronteras, no viendo en ello más que una fase de su propio marco evolutivo que habrá de ser estudiado con mayor detenimiento en el futuro. Recordemos, insistiendo en este planteamiento, que el Valle del Vinalopó tendrá ese carácter frontero a partir de la segunda mitad del siglo XIII, entre los reinos de Castilla y Aragón, momento en el que estas

(2) AZUAR RUIZ, R. «Excavaciones arqueológicas en el recinto amurallado denominado del Rio". Aspe. (Alicante. N.A.H. 15. 1983. p. 297-340.

LLOBREGAT, E. sLa antigua sede episcopal de Ilicitana". Festa d' ELx. Homenaje a Pedro Ibarra Ruiz. Alicante, 1978.

- -El origen romano de Elda. Alborada, XII. 1976.

REYNOLDS, P. .Cerámica Tardorromana modelada a mano de carácter local, regional y de importación en la Provincia de Alicante. Lucentum, IV. 1985. pp. 245-267.

GONZALEZ BL.ANCO, A. et Allii. -La ciudad hipsno-visigoda de Begastri. (Cabezo Roenas) Cehegín-Murcia. XVI C.N.A. Zaragoza, 1983. p. 1011-1022.

GONZALEZ BLANCO, A. -Yecla en los siglos de la Antigüedad Tardia. I Jornadas de Historia de Yecla. Homenaje a D. Cayetano de Mergelina. Yecla, 1986. p. 63-73.

RUIZ MOLINA, Liborio *El poblamiento rural romano en el área cle Yecla. (Murcia). Antigiledady Cristianismo, V. 1988. p. 565-598. 
fortalezas serán adaptadas a las nuevas necesidades militares, y por supuesto obedecerán a conceptos muy diferentes como estructuras organizativas de población viendo en lo sustancial la expresión del poder político señorial en buen número de los casos, y no el elemento colectivo de protección, que habían tenido hasta entonces.

Por tanto el hị̦n, desde un punto de vista morfológico debía obedecer a un núcleo de población instalado al abrigo de las defensas de un castillo, que permitía el refugio en casos extremos, siendo de menor entidad que la qal'a (fortaleza mayor) definida como ciudadela fortificada en el oriente musulmán y como alcalzaba en Al-Andalus, integrada en los sistemas defensivos del ejército.

Desde una óptica poblacional el hiș̣n contaba con menor importancia que la Madina (ciudad), aunque mayor que la qarya o balda (alquería o poblado). De la Madina podían depender desde un punto de vista administrativo, uno o varios hūsūn y varias alquerías, de igual forma que del hișn podían depender aquellos poblados rurales instalados en su territorio, siempre en función de su carácter dentro de la cora. (Distritos provinciales).

Levi Provençal describía el hisn en los siguientes términos: ‘... después de las alcalás, venían mucho más en número los castillos propiamente dichos (en árabe ḥiṣn). Esta palabra entraba a veces en composición con un topónimo puramente árabe como hiṣn al-lawz= Iznalloz, o hișn al-qasar= Aznalcazar; pero por lo común, el viejo nombre romano o ibérico subsistía sin modificación - hemos de precisar que Yakka no es un topónimo árabe, probablemente responde a un origen ibérico - El hișn, emplazado siempre en lugar elevado, o mejor en la cima de un cerro poco accesible estaba ante todo constituido por un sólido recinto que lo circuía, salvo que en algunos de los casos hubiera un tajo a pico. Tal muralla hecha de manpostería o de tapial estaba flanqueada por 
torres y fortines en los ángulos y tenía un camino de ronda y almenas. Casi siempre el recinto de la fortaleza no contaba más que con un espacio restringido, y naturalmente muy accidentado, sin más ingreso que una puerta de sólidas hojas, forradas de placas de hierro, y a veces precedidas de un puente levadizo, que permitía franquear el foso (jurdaq), si este reforzaba el sistema defensivo de los muros. Dicho limitado recinto no era más que un reducto capaz de resistir asaltos enemigos, $y$ soportar un asedio, y este reducto, llamado baram-al ḅișn, no contenia más que unos pocos instalaciones permanentes: cisternas para aguas pluviales, algún almacén de armas o de reserva de viveres, ciertos alojamientos elementales en los torreones y la torre del bomenaje. Por debajo y fuera del recinto, donde la cuesta era menos pronunciada, empazaba el arrabal (rabad) del castillo en el que vivian las gentes de la guarnición con su familias y los pocos artesanos y comerciantes que trabajaban en un mercadillo, a la sombra de la mezquita de modestas dimensiones.... (3).

Con las debidas matizaciones, esta descripción puede resultarnos válida en cuanto a la distribución espacial del hiṣn. Este se estructuraba en dos espacios: el albacar o área destinada al refugio de la población con sus enseres y animales; y el reducto principal donde se sitúan las instalaciones de carácter permanente: cisternas y depósitos de víveres y armas. Un tercer espacio asociado al castillo lo constituía el arrabal o núcleo de población permanente. La qarya o balda queda en cuarto grado asociativo, localizada en el territorio circundante, conformando lo que denominamos Iqlim o clima, con la acepción más puramente geográfica, o lo que es lo mismo, por buscar un símil actual, territorio o término municipal (4).

(3) LEVI PROVENÇAL Historia de España de Menendez Pidal. Madrid, 1957. T. V. p. 34-35.

(4) Este esquema se da en prácticamente todos los husun del área valenciana. Ver Bazzana, Cressier, Guichard. Op. Cit. 
Ibn al-Abbār en la Takmila, hace referencia a un personaje llamado Abū 'Amur Muhammad ben Muhammad... al-Lahmí, vecino de Murcia, cuya familia era originaria de Yakka (Yecla), añadiendo que ésta era uno de los distritos de Murcia (min a'māl Mursiya), si aceptamos, como así lo hacemos, las consideraciones de Alfonso Carmona al indicar que: "el término a'mal (plural a'mal) en su acepción geográfica-administrativa, estoy convencido de que designó algo así como lo que hoy recibe el nombre en España de "partido judicial». Me baso en que el sistema debió guardar probablemente analogía con el vigente en Marruecos hasta la época colonial. Allí al frente de cada uno de los distritos (amalatos les llamaban los africanistas españoles de principios de siglo) había un cadí -propuesto por el juez de la capital- quien a su vez podría designar personal con atribuciones a los jueces de paz de los distintos pueblos de la jurisdicción. La traducción literal de la expresión min a'māl Mursiya "es uno de los distritos de Murcia", parece evidenciar que significa que no solo pertenecían a Murcia, como parte de una de sus divisiones administrativas, sino que era capital de distrito o cabeza de partido judicial, ya que dicho 'amal recibía el nombre de Yakka (ḥiṣn), localidad que era por tanto su capital" (5).

Estas precisiones de Carmona González nos abren nuevas perspectivas en nuestros trabajos de investigación, rompiendo con la visión que teníamos de estos emplazamientos como pequeños núcleos rurales del interior de Murcia, con un relativo valor desde un punto de vista estratégico.

\section{II}

El hișn de Yecla se localiza en un cerro de formación calcárea situado a 38 37'Lat. $\mathrm{N}$ y 1 6' $\mathrm{Lg}$. W. El cerro cuenta con una altitud de $754 \mathrm{~m}$. sobre el nivel del mar, dominando una amplia vega a la que van a

(5) CARMONA GONZALEZ, A. -Yakka. Gumalla, Bilyana* Yakka, 3. Yecla, 1991. p. 15-21. 
desembocar las aguas de las diversas sierras del término municipal de Yecla: al Norte, la Sierra del Príncipe y Cuchillo; al Noroeste, Tobarrillas, Marisparza y Santa Bárbara; al Este la Sierra de Salinas y al Oeste las Moratillas y el Arabí.

Este valle-corredor, que recorre en dirección N-S la totalidad del territorio municipal, pone en contacto la ciudad de Yecla con la Meseta, SE y Levante peninsular. El cerro se sitúa en el centro de este eje, conformando el conjunto, a grosso modo, el Iqlim o clima de Yecla.

El ḥiṣn o castillo propiamente dicho cuenta en su cima con una planicie de unos $3.200 \mathrm{~m}^{2}$ a la altura de la curva de nivel $750 \mathrm{~m}$., a lo largo de la cual se desarrolla el recinto superior de la fortaleza árabe. Este nos describe una planta irregular con tres salientes donde se instalan tres torres defensivas, situadas en los extremos NE, NO y SE. Las dos primeras son de mayor envergadura, integradas en la línea de fortificación de la cara norte, que describe un trazado de cubos en saliente. La cara Sur y Este cuenta con menor obra defensiva, por la propia disposición natural del terreno.

Las dos campañas arqueológicas efectuadas en el yacimiento nos han permitido situar de forma provisional las fases de ocupación, indicando los porcentajes cerámicos más elevados los siglos XII al XIII, con una probable continuidad en los siglos XIV y XV.

Por el momento podemos distinguir dos áreas diferenciadas en cuanto a la distribución espacial: el albacar, con una extensión de $2 / 3$ sobre el total de la superficie, incluyendo la Torre NE y el reducto principal que ocuparía el espacio restante, situado en el extremo NO, donde se localiza una gran cisterna (6).

(6) RUIZ MOLINA, L. "El hisn rural de Yecla. Aportaciones a la Arqueologia Musulmana de la Región de Murcia en áreas del interiorn. Miscelanea Medieval, XVI. Murcia, 1990-1991. 237-266 p. 
Los restos del núcleo de población se extienden extramuros al pie del reducto principal entre las curvas de nivel 705 y $740 \mathrm{~m}$. en una superficie de unos $2000 \mathrm{~m}^{2}$ aproximadamente.

El territorio dependiente del hiṣn se estructura en pequeños núcleos de población, bien tipo qarya, balda o rahal, dedicados exclusivamente a actividades agrícolas y ganaderas, situándose probablemente en las antiguas instalaciones agrícolas romanas (villas rústicas), así por lo menos parecen evidenciarlo los restos arqueológicos de las "villae" de los Torrejones, Marisparza y el Pulpillọ (7).

En el siglo XII, las fuentes árabes nos dan noticias de Jumilla (Ğumalla), Yecla (Yakka) y Villena (Bilyāna), las dos primeras con la categoría de hișn y la última como madina. En los tres casos con el calificativo 'min a'māl Mursiya' (uno de los distritos de Murcia).

Joaquín Vallvé, en base a los textos del geógrafo al-Uidrī (m. 1085), nos presentaba los diecisiete distritos que formaban la Kura de Tudmir: -Lurqa (Lorca); Mursiya (Murcia); Al-Askar, Santiyiyala (Chinchilla); Ils (Elche); Iyih al-Sahl (Hellín); Bukasruh al-qal'a; Taibiliya (Taibilla); Tutitiya; Ibn al-yay'; otro Bukasruh; Mawroh; Balis (Vélez); Bayra (Vera); Tawtana (Totana); Laqwar (Alhama de Murcia) y Farqasa" (8). Esta división administrativa, quizá la vigente en los últimos tiempos del califato y el periodo de las taifas (s. X-XI), no informa de Jumilla, Yecla y Villena, lo que nos puede dar a entender una reestructuración admi-

(7) RLIZ MOLINA, L. -El poblamiento rural romano..... P. 596.

(8) VALLVE BERMEJO, J. -La división territorial de la España musulmana. (II). La Cora de Todmim. Al-Andalus, XXXVIII, 1972. 
nistrativa (amalatos) en el territorio murciano a finales del siglo XI y principios del siglo XII, momento en que estos lugares comienzan sin duda a tener cierto peso específico en el ámbito del SE. y Levante peninsular (9).

Nuevos datos nos van permitiendo fijar con mayor precisión los límites orientales de la cora de Murcia. El caso de Biar, incluido por Vallvé en la circunscripción de Murcia, debemos adscribirlo, por lo menos desde principios del siglo XII, como un hișn dependiente del distrito de Játiva (Satiba). Así nos lo indica Ibn al-Harrat (m. 1186) en su Ihtsar Iqtibas alauwar (10):

"Biyār (Biar) pertenece al distrito ('amāl) de gurgan (norte de Irán). Hay otro Biyār, perteneciente al distrito ('amāl) de Satiba (Játiva) en el alAndalus" (11).

Creo que es muy significativo el hecho de que el tratado de Cazola, firmado el 20 de marzo de 1179, entre Alfonso VIII de Castilla y Alfonso II de Aragón, en el que se delimitaban las áreas de expansión de ambos estados, se fijara en los siguientes términos:

"Valentiam et Totum regnum Valentiae, cum omnibus suis pertinenciis, heremis et populatis, que sibi pertinent et pertinent debent similitir laudat, codecit atque deffinit eidem et sucessoribus suis in perpetuum exativam (Satiba), cum omnibus sibi pertinentibus, heremis et populatis, et totam terram, heremam et populatam qui est a portus ultra Biar,

(9) Alfonso Carmona sobre el topónimo Bilana/Billana atribuido de forma errónea a Villena, como una de las ciudades incluidas en la Capitulación de Todmir, da una aclaratoria explicación al respecto en «Yakka, Gumalla, Bilyana. Yakka, 3. 1991. p. 15, demostrando que Villena, al igual que Jumilla y Yecla comenzarán a tener importancia a partir del siglo XII.

(10) IBN AL-HARRAT Ibtisar Iqtibas al-anwar. Ed. E, Molina / J. Belchí.

(11) Debo la traducción a Alfonso Carmona González, que considera válido este dato para la primera mitad del siglo XII, puesto que Al-Harrat, extracta la obra de Ar-Rusti (m. 1147). 
qui portus dicitur portus Biar, sicut reciput versus Exativam et Valentiam; et Deniam et totum regnum Denierum omnibus suis pertinenciis, heremis et populatis, sicut tendit et ducis portus usque admareet vadis usque Calpn (12).

Años más tarde, el tratado de Almizra, firmado el 26 de marzo de 1244 por Jaime I de Aragón y el Infante D. Alfonso de Castilla, fija las líneas fronterizas entre ambos estados respetando el espíritu de Cazola, marcándose como punto inicial la costa de Villajoyosa, dejando por tanto Alicante en territorio murciano, para tomar dirección Oeste hacia Bussot, el pico de Maigmó, y tomar allí la referencia el valle del Vinalopó, para continuar por el valle de Ayora.

Veintidós años después de Almizra, el privilegio concedido por Alfonso X de Castilla para el establecimiento de los límites territoriales del obispado de Cartagena, viene a confirmar la frontera de Murcia, que coincidirá con la nueva demarcación eclesiástica:

"Como los avía ante que la guerra de los moros commenccasse que movio contra nos el rey de granada. (Se refiere a las sublevaciones mudejares). Et los terminos son estos, Alicante (Laqant) con su término, assi como parte del término con el Rey de Aragón, Petrel (Batrir) et Sax (Says) et Villena (Bilyana o Bilana), la tierra de don Manuel (aquí entraría Yecla), nuestro hermano, coparte de las tierras de Aragón, et el val de Ayora con Cofrentes, et otrossi como parte de la tierra de Aragon, et Jorquera con su termino, et con la tierra de Goncalo Royz de Atienca, et Chinchilla (Yinyalla) con su término..." (13).

(12) GONZALEZ, J. El reino de Castilla en época de Alfonso VIII. Madrid, 1960. II. p. 529.; también en Joaquín Vallvé Bermejo. "La división territorial en la España musulmana..." p. 150.

(13) TORRES FONTES, J. Colección de documentos para la bistoria de Murcia. I. Documentos de Alfonso X el Sabio. Murcia, 1963 p. 37. 
Pensamos por tanto, como muy probable, que descle la declaración de intenciones de Cazola en el último cuarto del siglo XII, hasta el tratado de Almizra y la constitución territorial del obispado de Cartagena, se sigue la línea que marcaba los límites territoriales entre las coras de Valencia y Murcia (Aragón y castilla), vigentes al menos durante todo el siglo XII y la primera mitad del siglo XIII. Las palabras de Ibn Sa'ïd a su paso por las tierras de Murcia en 1241 camino de Alicante, son un punto de apoyo más a lo que aquí sostenemos:

“Cuando viajé desde Murcia al mar pasé por Orihuela [...], y por la ciudad Alicante que es el puerto de Murcia, la gente se hace a la mar desde allí para ir a Ifriquiya [...], nunca vi en el al-Andalus ciudadela más inexpugnable.... (14).

En el año 1327, Jumilla realiza una escritura de amojonamiento y deslinde del término Municipal a fin de "aclarar e determinar los terminos e moiones e poços e aquas de la dicha villa de Jumilla siendo por ello llamadas a su pedimiento e uno con el en la dicha villa los procuradores de los concejos de la comarca primeramente de Chinchilla e Hellín e Cieça e de Ricote e de Molina e de Murcia e de Havanilla e de Chinosa e de Monovar e de Yecla e de Montealegre e con el señor de la alqueria de Ontur y Albatana et todos los cuales dichos procuradores de los dichos concejos e logares fueron a determinar e determinaron lo sobre dicho en uno con mosen Priete, alcaide del castillo de la dicha villa el cual fue por hombre del dicho senyor don Goncalo en presencia de mi dicho notario, los cuales declararon ciertas ordenanças en esta manera que sigue: primeramente fue declarado por los dichos procuradores de los dichos concejos $e$ Aljamas e alcaides en uno con el dicho don Gonçalo....

(14) CARMONA GONZALEZ, A. *Noticias geográficas árabes referentes de la Bilad Tudmir.. Murgetana, 72 Murcia, 1983 p. 118.

- -las Vías de comunicación en época árabe. Caminos de Murcia. Murcia, 1988. p. 156. 
Es importante hacer notar la participación de los 'viejos de las aljamas' en la elaboración de la carta de amojonamiento. Por una parte denota el peso específico con que aún contaba la población musulmana entrado ya el siglo XIV; por otra parte se observa como ésta conserva aún sus viejas estructuras organizativas, y lo que quizá pueda interesarnos más es que en cierto modo se les pide asesoramiento en la demarcación de los límites territoriales, como buenos conocedores del terreno. No hay dudas de que en el caso del amojonamiento de Jumilla en poco debió variar con respecto a los antiguos límites del Iqlim musulmán de los siglos XII y XIII. De la totalidad de poblaciones implicadas solo aparecen los representantes de las aljamas de Ricote, Abanilla, Monovar y Chinosa, lo que nos muestra las áreas de mayor densidad de población mudejar en las comarcas próximas a Yecla, añadiendo a ellas el Valle de Ayora y en menor medida el Valle del Vinalopó (15).

Los límites territoriales de Jumilla y Yecla quedaban fijados de la siguiente forma: aItem fueron determinados los mojones e termino de entre Yecla y Jumilla estando presente el procurador del concejo de la dicha villa en esta guisa començando del dicho pino rasposo de cara a la Sierra del Carche, luego a la foya de dicho Carche mesmo a la alber(ca) grande es el otro moion, et luego pasando a la Sierra del buey (en) el collado ahi esta el otro moion et dende a la Macolla cerca del camino que va (de) Jumilla a Yecla buen rato encima del algibe esta el otro moion e el otro pasando el puerto que dicen de Fferran Garçia e donde va el otro moion al Barranco de los Gavilanes e dende el Morteruelo et aqui se acaban los moiones de Yecla." (16).

(15) BARCELO TORRES, Maria. Minorias islämicas en el País Valenciano. Historia y Dialecto. Valencia, 1984. Sobre el poblamiento mudejar es muy interesante el capitulo dedicado a conomía y sociedad. p. 62-105.

(16) LOZANO PEREZ, J. M. Carta de amojonamiento del término de Jumilla. Jumilla, 1982, p. 62-64. 


\section{IV}

El estudio de los itinerarios o vías de comunicación de acuerdo a la información que las fuentes escritas árabes nos ofrecen, junto con el complemento aportado por los documentos cristianos, ha de considerarse como elemento de primer orden en cualquier intento serio de hacer geografía histórica.

De Yecla sólo contamos con la noticia de su distancia con respecto a la capital de la cora, ofrecida por el biógrafo Ibn Dihya (m. 1235) en su aclaración sobre la procedencia del apellido al-Yakki, anotando que es originario de Yakka población «a 45 millas al norte de Murcia" (17).

Un problema de difícil solución es el dar un valor uniforme a la milla. Tomemos como base el itinerario de al-Udrī que unía las ciudades de Cartagena con Chinchilla (s. XI): (Entre paréntesis presentamos las distancias actuales en $\mathrm{Km}$ ).

"De Cartagena a Murcia, 30 millas $(53 \mathrm{Km})$. Hasta Molina 8 millas $(12 \mathrm{Km})$ A Cieza 25 millas $(29 \mathrm{Km})$. A Minateda (Madinat Iyih) hay 30 millas $(40 \mathrm{Km})$. A continuación 10 hasta Tobarra $(14 \mathrm{Km})$. Luego hasta Chinchilla, 35 millas. (42 Km)» (18).

La equivalencia de la milla oscilaría, teniendo en cuenta las distancias actuales, entre $1160 \mathrm{~m}$. y $1760 \mathrm{~m}$., lo que se traduciría en el caso de Yecla de $52.200 \mathrm{Km}$ a $79,200 \mathrm{Km}$. la distancia que la separaba de Murcia. Las rutas actuales cubren la distancia de $98 \mathrm{Km}$., vía Jumilla, y $84 \mathrm{Km}$. vía Pinoso-Fortuna. Ambas quedan fuera de la banda kilométrica expuesta,

(17) CARMONA GONZALEZ, A. •Yakka, Gumalla, Bilyana..* p. 18.

(18) CARMONA GONZALEZ, A. .Vias de comunicación en época árabe...* p. 156-158. Se presenta un preciso análisis descriptivo de la ruta Cartagena-Murcia y sus conexiones con la ruta CórdobaValencia. 
aunque la última es la que más se aproxima. Podría pensarse en otra ruta alternativa a las expuestas y en este sentido se ha sugerido que el paso podría efectuarse por la Sierra del Carche, hacia el Oeste, a unos $10 \mathrm{Km}$. de la actual ciudad de Jumilla, para desde allí enlazar con el trazado de al-Udri, a la altura del puerto de La Losilla (19). La distancia de este recorrido es aproximadamente de 80 a $82 \mathrm{Km}$. por lo que caería dentro de las posibilidades kilométricas fijadas. Desconozco la argumentación que sostiene esta propuesta, aunque a mi juicio no parecen existir indicios sólidos que evidencien la utilización de esta ruta como enlace habitual de Yecla con la capital de la cora.

Lo procedente en estos momentos, a falta de prospecciones arqueológicas con mayor rigurosidad, es exponer lo que conocemos por la documentación escrita sobre los itinerarios y caminos en la Edad Media, que a buen seguro será un consistente punto de partida para futuros trabajos. Así pues, en primer término analizaremos las rutas principales de época musulmana en el ámbito de la región, para poder luego apuntar las posibles conexiones de Yecla con éstas, en base también a los escasos datos con que contamos.

La ruta que unía Cartagena con Chinchilla ofrecida en los textos de AlUdrī y que consideramos vigente al menos en los siglos $\mathrm{X}$ y $\mathrm{XI}$ ya ha sido expuesta con anterioridad. El mismo autor presenta una segunda ruta que ponía en contacto Murcia con Valencia por el interior, aunque de forma menos detallada que la primera y marcando las distancias entre los distintos emplazamientos en jornadas o etapas. Según Alfonso Carmona la jornada o etapa podría oscilar entre "30 y 60 kilómetros, dependiendo de si se viajaba a pie o a montura, de la estación del año y de la naturaleza del terreno. El camino recorrido en una jornada dependía también en buena

(19) CARMONA GONZALEZ, A. -Yakka, Gumalla, Bilyana... p. 18. Se hace referencia textual a esta ruta propuesta por F. Muñoz López, del trabajo inédito .El castillo de Yecla y el poblamiento musulmán en su comarcam. 
medida de la distancia a la que se encontrase el lugar que se considerase idóneo para pernoctar - este final de etapa era un Manzil, 'venta o pasada'“ (20). El trayecto en cuestión quedaba de la siguiente manera: "De la ciudad Murcia a Orihuela una etapa (20 Km. aprox.) hasta el pueblo de Aspe otra jornada $(36 \mathrm{Km}$.); otra jornada hasta Biar $(44 \mathrm{Km}$.); hasta la localidad de Játiva otra $(60 \mathrm{Km}$.) de allí a Alcira una etapa y otra hasta Valencia.» (21).

Si comparamos este itinerario con el señalado por al-Idrīsī (m. 1171) un siglo después, observaremos un mayor detalle en la descripción y la fijación de las distancias en millas. Dice al-Idrîsĩ: «De Murcia a Orihuela hay 12 millas $(20 \mathrm{Km}$ ); de Orihuela a Elche, 18 millas $(32 \mathrm{Km}$.), al hiṣn de Crevillente, 18 millas (24 Km.), a Biar 35 millas ( $46 \mathrm{Km}$.); a Játiva 35 millas (60 Km.), a la ciudad de Valencia 36 millas...n. El mismo itinerario nos lo ofrece el autor con mayores precisiones: “De Murcia a Orihuela hay doce millas $(20 \mathrm{Km}$.). De esta localidad a Albatera hay seis millas. $(13 \mathrm{Km}$.): Allí es donde está el parador (manzil). De esta al hişn de Aspe hay seis millas. (23 Km.), luego se dirige uno al castillo de la Mola que está a orillas del río de Elche; entre Aspe y la Mola hay seis millas $(6 \mathrm{Km}$.). El camino lleva luego hasta Petrel (Bitrir), donde se completa la etapa que es de 35 millas. (La distancia actual de la Mola a Petrel es de $12 \mathrm{Km}$. aproximadamente), y luego al hișn de Onteniente (Untinyan). Desde allí a la ciudad de Játiva hay 30 millas $(29 \mathrm{Km})$. . (22).

Lo primero que salta a la vista, y que ya apuntábamos anteriormente, es la falta de uniformidad en las equivalencias de las millas en relación con las distancias kilométricas actuales. Por otra parte, el primer texto parece desviar la ruta a la altura de Crevillente, citando Elche que queda a la derecha del itinerario. Más que un error de la transmisión del texto

(20) CARMONA GONZALEZ, A. "Viàs de comunicación en época árabe" p. 153-154.

(21) Ibidem. p. 159.

(22) CARMONA GONZALEZ, A. "Yakka, Gumalla, Bilyana...” p. 16-17. 
como apunta el profesor Carmona, veo un intento de dar mayor información al viajero, indicando la existencia de una ciudad importante (Elche), como punto intermedio en la ruta de Murcia a Alicante. Las distancias dadas por al-Idrisī desde Orihuela a Crevillente y desde la primera a Elche es la misma, 18 millas; sin embargo las distancias actuales son $24 \mathrm{Km}$. a Crevillente y $32 \mathrm{Kms}$. a Elche, lo que puede sugerir un camino diferente hasta Alicante, que partiría de Orihuela, vía Callosa-Catral-Elche. Otro dato que nos parece significativo es la exclusión de Villena en esta ruta, sabiendo como sabemos que en el siglo XII era una población de cierta importancia a juzgar por las referencias que ibn al-Abbār nos hace de ella: "Abū 'Abdallāh Muhammad ben Aḥmad [...], de Jaén [...] Ibn'Iyyãd, que transmitió tradiciones que aprendió de él dice: Lo conocí en Bilyāna (Villena) [cabeza de] uno de los distritos de Murcia (min a'amāl Mursiya); creo que había tenido que salir de su tierra con motivo de los sangrientos conflictos (fitna) que estallaron en época de la caida del régimen de los lantūmíes. [almorávides] (23).

Al-Idrīsī al tratar la ruta Murcia-Chinchilla lo hace de forma sucinta, hasta el punto de no citar ni tan siquiera las etapas, señalando única y exclusivamente, que desde Murcia a Chinchilla había 50 millas, lo que cae en contradicción con la distancia marcada por al-Udri entre ambos puntos, con un total de 108 millas. Esto nos muestra que éste camino había dejado de tener la importancia de otros tiempos, por lo menos desde el siglo XII. Recordemos que la conexión con Toledo había quedado rota ya que esta ciudad estaba en manos castellanas, de hecho el propio al-Idrīsĩ indicaba que desde Chinchilla el itinerario se dirigía a Cuenca. De la misma forma, la ruta de Murcia a Córdoba no debía ser muy frecuentada, si nos atenemos a la información que se nos ofrece: "De Murcia a Córdoba hay diez etapas» (24).

(23) Ibidem. p. 17.

(24) CARMONA GONZALEZ, A. Vias de comunicación den época árabe» p. 162. 
Parece pues, no quedar dudas de que durante la segunda mitad del siglo XII y la primera mitad del siglo XIII, la ruta Valencia-Andalucía (Almería): Valencia, Onteniente, Biar, Petrel, Aspe, Crevillente, Albatera, Orihuela, Murcia, Alcantarilla, Librilla, Alhama, Lorca pozos de ar-Rabatta, Vera Mojácar, La Rabita y Almería, debió potenciarse en gran medida, lo que propició el desarrollo de comarcas próximas a la misma, casos estos de Jumilla, Yecla o Villena. Un dato que parece confirmarnos lo dicho lo encontramos en referencia a la localidad de Aspe, que en el siglo XI (alUdrī) figuraba como una simple qarya (poblado) y un siglo más tarde (alIdrīsī) se nos muestra con el grado de hịsn.

Las fuentes cristianas pueden igualmente aportarnos datos complementarios a los itinerarios de gran utilidad. Ya señalábamos cómo en la carta de amojonamiento de Jumilla (1327) figuraba el llamado puerto de Fferran García, situado en el camino que unía a la villa de Jumilla con la de Yecla, correspondiendo al actual puerto de Jumilla. Este paso hemos de suponer que estaba abierto al menos desde finales del siglo XIII, adquiriendo una relativa importancia en el siglo XVI, como paso obligado entre Granada y Valencia. (Vía Huéscar-Caravaca-Calasparra-Jumilla-Caudete-Fuente la Higuera) (25).

El nuevo reparto territorial y fijación de fronteras entre Castilla y Aragón, efectuado con los acuerdos de Torrellas-Elche, (1304-5) daban a Yecla, por primera vez en su historia, un carácter frontero entre ambos estados: "que del subirano logar del termino de Villena de parte del termino de Almansa, e otrossy del subirano veçyno termino de Alcaudet que parte termino con Almansa e con Pechín. El otrossy de subirano señor de Jumilla departe término con Ontur e con Tobarra en con Fellín e con Cieça, todos los logares que son dentro de estos moiones fasta las pates

(25) MOLINA MOLINA, A.; SElVA INIESTA, A. -Los caminos murcianos en los siglos XIII-XVI. Caminos de la Región de Murcia. Murcia, 1989. P. 173-174. Se recoge detalladamente el llamado .Camino de Los Valencianos. 
de las tierras de Aragón sean en su jurisdiçion, salvo el de Yecla con todos sus terminos que finque libre e quito a don Juan Manuel e de juricdiç̧ion del Rey de Castilla.." (26).

Solventada la guerra civil entre Pedro el Cruel y Enrique de las Mercedes, en favor de este último coronado como Enrique II de Castilla, cedió las antiguas tierras del Señorío de los Manuel, con grado de Marquesado de Villena, a Alfonso de Aragón, fiel aliado durante la guerra. Aurelio Pretel Marín afirmaba a tal respecto: "Aunque los pueblos del Marquesado se resistieron, al fin hubieron de aceptar por señor a don Alfonso ( 1372-1395), no sin hacerse confirmar por él todos los privilegios y costumbres de época de los Manuel. A pesar de su carácter autoritario, que no tardaría en poner de manifiesto, don Alfonso supo comenzar su mandato recogiendo la obra de don Juan Manuel y sirviéndose de ella para aumentar su propio poder, la integración de sus estados y la independencia con respecto a la Corona, con él las tierra de la Mancha de Montearagón llegaría al culmen de su autonomía, respecto del Rey de Castilla, cuyas órdenes serán sistemáticamente desoídas y cuyos súbditos de los reinos de Toledo o Murcia recibirán aquí el mismo trato de extraños que los aragoneses desde Murcia en las Aduanas de Almansa o Yecla." (27)

La importancia de la aduana de Yecla como punto fronterizo entre Castilla y Aragón debió comenzar a principios del siglo XIV, alcanzando su máximo desarrollo durante el siglo XVI, momento en que aún mantenía ese carácter frontero, por lo menos así lo refleja la Relación de Yecla, fechada en 1575, (Relaciones Topográficas de Felipe II):

¿CAPITULO 5. Y si es pueblo que está en frontera con algún reino extraño que tan lejos está de raya y si es entrada o paso para él o puerto o aduana do se cobren algunos derechos.

(26) LOZANO PEREZ, J. M. Carta de amojonamiento... p. 83.

(27) PRETEL MARIN, A. Breve bosquejo bistórico del Señorio de Villena y sus Instituciones. 1980. p. 6. 
Respuesta: a los cinco capítulos dixeron e hiçieron relaçion que la dicha villa de quien se da la dicha relaçion esta situada en frontera del reino de Valençia y departe terminos con la villa de Caudete que es del dicho reino de Valençia y los mojones y departimientos de los dichos terminos estan a una legua de esta dicha villa y que por otra parte confina los terminos de la dicha villa de Yecla con terminos de la villa de Monovar que es del reino de Valençia y por esta parte hay tres leguas desde esta villa a dichos términos de la villa de Monovar y que esta dicha villa de Yecla es paso y aduana para entre los reinos de Castilla y el de Valencia y Aragón y otros estraños y en ellos se cobran derechos devidos a su Majestad de todos los generos de mercaderías que por él pasan, así de los que van a estos reinos estraños, como de los que vienen de estos reinos de Castilla.. (28)

Si atendemos a la demarcación territorial de Marquesado de Villena propuesta por Pretel Marín, es fácilmente observable que los pasos fronterizos con Valencia se efectuaban a través de los términos municipales de Caudete y Monóvar, ya que los límites hacia el SE. se marcaban con los propios límites territoriales de Almansa, Villena y Sax.

La ruta ganadera que ponía en contacto la serranía de cuenca con los pastos murcianos, y que se efectuaba a través de Jorquera, Chinchilla, corral Rubio, Montealegre del Castillo, Yecla, Pinoso y Fortuna, aparece referenciada en la sistematización y uniformidad realizada por la Mesta sobre los montazgos en el año 1380 (29). La conquista de Murcia favoreció a los ganaderos de Cuenca que veían así ampliados sus pastos de invierno. La Cañada Real de los Serranos a su paso por Yecla uentra procedente de Montealegre, le cruza el arroyo de agua salada o la Rambla de los Hitos, se

(28) Hemos utilizado la transcripción de J. Blazquez Miguel en Yecla en tiempos de Felipe II. Yecla, 1981. p. 24. Para conocer la actividad de la Aduana de Yecla en el siglo XVI en la misma obra p. 78 .

(29) KLEIM, J. La Mesta. 2 Ed. Madrid, 1981. p. 206. 
ensancha a $180 \mathrm{~m}$. en una longitud de $1200 \mathrm{~m}$., terminando este ensanche donde le entra por su derecha el camino de Madrid. En el Pulpillo, a su derecha, se le une el cordel del Pozuelo o de Andalucía, recorriendo juntos $600 \mathrm{~m}$., y al separarse hay un aguadero concejil dentro de ambas vías pecuarias. La cruza la travesía de Jumilla a Caudete y el camino de Madrid. Cruza la Rambla del El Pulpillo, y se abre en un descansadero, estando dentro de él la Fuente Principal del pueblo (Cerro de la Fuente) y en nacimiento de Bermudes. Cruza la Carretera de Jumilla, y el cordel de la Flor, deja a su izquierda el cementerio y le cruza el camino de Murcia. A continuación se ensancha progresivamente llegando a tener $450 \mathrm{mts}$. de ancho, y continuando con $120 \mathrm{~m}$. hasta la Balsa Nueva, que se encuentra dentro de la vía pecuaria, teniendo este abrevadero forma cuadrada, de unos 280 m. de lado. De aquí arranca el cordel de la balsa nueva, que sigue con anchuras variables superiores a $100 \mathrm{~m}$., por caserío de Dedos, Caserío de los Calderones, y el paraje del Portichuelo. Le cruza la carretera de Murcia, la Rambla de los Puntales y la Rambla de la cima de la Beata o de los Estrechos, se ensancha en un recorrido de $700 \mathrm{~m}$. de anchura de $140 \mathrm{~m}$. de longitud, cruza el camino de los Estrechos y después el Barranco de las Peñas y el Barranco de la casa de Mosen y sale de este término, dejando a la izquierda el cerrico del Aguila, para continuar por el de Pinoso, de la provincia de Alicante. La longitud del recorrido descrito es de $39 \mathrm{kms}$. y la anchura legal de esta vía pecuaria es de 75,22 mts., excepto donde se ensancha que es superior" (30). En tierras alicantinas y por el denominado Plá del Castellá (o Llanos de Castilla), se introduce por Pinoso buscando Abanilla y Fortuna. Desde los Llanos de Castilla queda asegurada la conexión con el Medio Vinalopó (Elda, Monóvar, Novelda, Aspe).

A lo largo del trayecto de la Cañada Real se observan una serie de construcciones hidráulicas relacionadas con la ruta ganadera. Nos referi-

(30) La descripción ha sido tomada de la Clasificación de Vías Pecuarias realizada en 1974, recogida por Cesar Flores Casanova y Carmen Flores Flores en -Vias Pecuarias de la Región de Murcia-. Caminos de la Región de Murcia. Murcia, 1989. p. 285. 
mos a los aljibes cimbrados, que en número de dieciséis componen el conjunto. Las características constructivas son similares a los estudiados por Lorenzo Cara Barrionuevo y Juana $\mathrm{M}$. Rodríguez López en el área del antiguo Reino de Granada: embalse o depósito por lo general de planta rectangular y subterráneo, una bóveda de medio cañón por aproximación de hiladas de piedras unidas con argamasa de cal y arena (cimbra), y una entrada de agua. Una puerta adintelada en uno de sus extremos para sus acceso al interior. La balsa o píleta de decantación para la depuración de las aguas de lluvía, sobresaliendo de la superficie la cubierta y los extremos de la nave (31).

Cronológicamente estas cisternas o aljibes podrían situarnos entre finales del siglo XII y principios del siglo XIII (período almohade), momento en el que los califas Yakub al-Mansur y Mahammad al-Nasir potencian una política de desarrollo hidráulico, promoviendo este tipo de construcciones, con la intención de favorecer las rutas terrestres más importantes, lo que en el caso de Murcia proporcionará desde mediados del siglo XIII una inmejorable infraestructura para la ampliación de las rutas ganaderas de La Mesta en estas tierras (32).

Así pues, considero que la Cañada Real de los Serranos debió ser esencial en el despegue económico de Yecla desde mediados del siglo XII, pues no sólo permitía el contacto con Murcia, vía Pinoso-AbanillaFortuna, sino que posibilitaba la comunicación, a través de MonóvarNovelda, con la ruta Murcia-Valencia descrita por al-Idrīsi, y por lo tanto la salida al mar, 'al puerto de Murcia', Alicante.

(31) CARA BARRIONUEVO, Lorenzo; RODRIGUEZ LOPEZ, Juana María. «El pastoralismo en el AlAndalus. Aproximación arqueológica en el SE. peninsular. Arqueologia X, 96. 1989. p. 40-48.

(32) RUIZ MOLINA, L; AZORIN CANTO, M. «Aljibes cimbrados en el área de Yecla (Murcia). Notas para el estudio de la ganadería transhumante en el NE de la Región de Murcia. Siglos XII-XIX= $I$ Coloquio de Historia y Medio Físico. Almería, 1989. p. 609-627. 
Un documento también de interés para el estudio de las comunicaciones de Yecla en época medieval, aunque tardío, nos lo presenta Juan Torres Fontes en su estudio sobre Yecla en tiempos de los Reyes Católicos. Se trata del litigio mantenido por los concejos de Villena, Sax y Yecla con el comendador del Señorío de Montealegre, que había cercado las dehesas donde tradicionalmente pastaban los ganados de estas poblaciones. Además pretendia modificar el trazado de la Cañada Real, desviándolo por el propio casco urbano de Montealegre. Lo que aquí nos interesa es un párrafo de la carta de los Reyes Católicos dirigida al doctor Barrientos, Corregidor de Murcia, en la que se le solicitaba información sobre esta queja, fechada el 29 de abril de 1497, donde se indicaba: «Sepades que por parte de los concejos, alcaldes, regidores e omes buenos de las villas de Villena, e Yecla e Sax, nos es fecha relaçion por su petiçion diziendo que teniendo los vezinos de las dichas villas derecho de ir por un camino que llaman camino viejo que va a la cibdad de chinchilla, por cerca de un lugar que dizen Montealegre, ...* (33). El camino viejo al que se hace referencia en el caso de Sax, corresponde en la actualidad con el denominado "camino de Sax", que une esta población con Yecla a través del Puerto de la Harina. Este trayecto, que en Yecla se le conoce popularmente como "camino viejo de los Romanos" confluía en la Cañada Real a la altura de la Fuente Principal, para proseguir en dirección a Montealegre. En el caso de Villena, próxima a la población de Sax, es probable que conectara con Montealegre por un trazado más corto, hacia la población de Caudete, siguiendo la antigua calzada romana que discurría por la "Traviesa de Caudeten, al pie de la Sierra de Santa Bárbara y los parajes de Tobarrillas y Marisparza, (estos últimos en el término municipal de Yecla), desembocando en la Venta de los Hitos, punto donde se enlaza con la Cañada Real (34).

(33) Para más detalles sobre el pleito en Juan Torres Fontes. Yecla en el reinado de los Reyes Católicos. Yecla, 1954. p. 21-22.

(34) RUIZ MOLINA, L.; MUÑOZ LOPEZ, F. .Las vias romanas de comunicación en la comarca de Yecla. (Murcia). Symposium Vias Romanas del SE. Murcia, 1986 p. 67-74.

BROTONS YAGUE, F. et allii -El tramo viario de Montealegre a Fuente la Higuera. Symposium Vias Romanas del SE. Murcia, 1986. p. 75-83. 
La utilización de estos dos pasos en época musulmana parece evidente si nos dejamos llevar por la intuición, aunque no sepamos con que intensidad. Por el momento no contamos con datos fehacientes que apoyen lo que no es más que una posibilidad.

\section{$\mathrm{V}$}

Estos apuntes son fruto de los trabajos arqueológicos de campo, iniciados hace dos años, y del estudio de las fuentes escritas árabes desarrollado durante el primer curso de doctorado del Departamento de Prehistoria, Arqueología, Historia Antigua e Historia Medieval de la Facultad de Letras de la Universidad de Murcia. Para su elaboración he encontrado el apoyo inestimable de D. Alfonso Carmona Gonzalez, Profesor Titular de Estudios Arabes de la Universidad de Murcia.

Igualmente he tenido un excelente complemento en D. Juan Torres Fontes y D. Miguel Ortuño Palao que con sus aclaraciones y sugerencias han hecho posible la gestación de éstas páginas. 